\title{
Cooper Pair in Two Nonstandard Situations
}

\section{P. Wróbel, Z. Starypan, J. Kaczmarczyk and J. SpaleK*}

Marian Smoluchowski Institute of Physics, Jagiellonian University

Reymonta 4, 30-059 Kraków, Poland

\begin{abstract}
We summarize briefly our recent work on the bound states of two electrons (Cooper pairs) in two nonstandard situations: when the quasiparticle masses depend on spin and when an electron pair is localized on a two-dimensional quantum dot and placed in an applied magnetic field. In both cases we are dealing with an extension of the original Cooper approach. The applicability of the results is briefly mentioned.
\end{abstract}

PACS numbers: 74.20.- $\mathrm{z}, 74.25 . \mathrm{Jb}, 71.10 .-\mathrm{w}$

\section{Introduction}

The concept of Cooper pair of electrons [1] was a starting point of the Bardeen, Cooper, and Schrieffer (BCS) theory of superconductivity [2], as the binding is mediated by coupling to the lattice which overcomes the repulsive Coulomb interaction between them. In recent years, this mechanism of pairing is being questioned for high-temperature and heavy-fermion superconductors, where a concept of real space pairing due to electron correlations induced by strong (short-range part) of the repulsive interaction is regarded as instrumental in producing the condensate of pairs [3]. Also, the binding of electrons into pairs on meso- or nanoscales [4] provides an opportunity to detect individual Cooper pairs in direct space, particularly in planar systems.

In this paper we summarize the recent effort of our group in studying electron pairs in various situations. Namely, we consider first a single pair in the spin-singlet configuration in a two-dimensional quantum dot (modelled by a twoparticle attractive potential well). We discuss also the binding of the pair in the presence of an applied magnetic field. In the second part, we consider the Cooper pair when the particle mass depends on its spin direction. This type of situation appears in the correlated narrow band system close to the Mott-Hubbard localization [5]. Both examples have been selected on the basis of our current interest in these novel systems.

*corresponding author; e-mail: ufspalek@if.uj.edu.pl 


\section{Bound pair in a planar system}

We consider first a system of two electrons in confining them planar attractive pairing energy

$$
V(r)=\left\{\begin{array}{rc}
-V_{0} & \text { for } r \leq a, \\
0 & \text { for } r>a,
\end{array}\right.
$$

where $2 a$ is the spatial extension of the potential. Hamiltonian of such a two-dimensional system of two electrons placed in a uniform applied magnetic field has the form

$$
H=\frac{1}{2 m}\left[\left(-\mathrm{i} \hbar \nabla_{1}-e \boldsymbol{A}_{1}\right)^{2}+\left(-\mathrm{i} \hbar \nabla_{2}-e \boldsymbol{A}_{2}\right)^{2}\right]-\left(\boldsymbol{\mu}_{1}+\boldsymbol{\mu}_{2}\right) \cdot \boldsymbol{B}+V\left(\boldsymbol{r}_{1}, \boldsymbol{r}_{2}\right),
$$

where the indices 1 and 2 correspond to the coordinates of the electrons, respectively. The quantity $\boldsymbol{\mu}=2 \mu_{\mathrm{B}} \boldsymbol{s}$ is the electron magnetic moment (with a minus sign, so it has the spin direction). Eigenvalues of the total spin $\boldsymbol{S}=\boldsymbol{s}_{1}+\boldsymbol{s}_{2}$ are $S=0,1$. Also, $S_{z}=s_{1}^{z}+s_{2}^{z}=0, \pm 1$, whereas $m$ plays the role of the effective mass if the system is immersed in a medium.

For the applied field oriented perpendicularly to the system plane, the rotational symmetry of the problem allows for the choice of the symmetric gauge for the vector potential $\boldsymbol{A}=(-y, x) \boldsymbol{B} / 2$, where $\boldsymbol{B}$ is along the $z$-axis. Introducing the center of mass and the relative coordinates, defined as

$$
\left\{\begin{array} { l } 
{ \boldsymbol { R } = \frac { \boldsymbol { r } _ { 1 } + \boldsymbol { r } _ { 2 } } { 2 } } \\
{ \boldsymbol { r } = \boldsymbol { r } _ { 1 } - \boldsymbol { r } _ { 2 } }
\end{array} \Longrightarrow \left\{\begin{array}{l}
\boldsymbol{r}_{1}=\boldsymbol{R}+\frac{\boldsymbol{r}}{2} \\
\boldsymbol{r}_{2}=\boldsymbol{R}-\frac{\boldsymbol{r}}{2}
\end{array},\right.\right.
$$

we separate Hamiltonian (2) into centered-mass and relative motion parts, which in the polar coordinates $\boldsymbol{R}=(R, \Phi)$ and $\boldsymbol{r}=(r, \varphi)$ read

$$
\begin{aligned}
& H=-\frac{\hbar^{2}}{2 M}\left[\frac{1}{R} \partial_{R}\left(R \partial_{R}\right)+\frac{1}{R^{2}} \partial_{\Phi}^{2}\right]+\frac{M \omega_{\mathrm{c}}^{2}}{8} R^{2}-\frac{\mathrm{i} \hbar \omega_{\mathrm{c}}}{2} \partial_{\Phi} \\
& -\frac{\hbar^{2}}{2 \mu}\left[\frac{1}{r} \partial_{r}\left(r \partial_{r}\right)+\frac{1}{r^{2}} \partial_{\phi}^{2}\right]+\frac{\mu \omega_{\mathrm{c}}^{2}}{8} r^{2}-\frac{\mathrm{i} \hbar \omega_{\mathrm{c}}}{2} \partial_{\phi}-2 \mu_{\mathrm{B}} B \hat{S}_{z}+V(r),
\end{aligned}
$$

where $M=m_{1}+m_{2}=2 m$ and $\mu=m_{1} m_{2} /\left(m_{1}+m_{2}\right)=m / 2$ are respectively the system and the reduced masses, and $\omega_{\mathrm{c}}=|e| B / m$ is the cyclotron frequency for a single electron (in SI units).

\subsection{Noninteracting pair: a summary}

In the absence of the pairing potential the eigenvalues and eigenfunctions take the form [6]:

$$
\begin{aligned}
& \left(E_{0}\right)_{N L n l}^{S, S_{z}}=\hbar \omega_{\mathrm{c}}\left(N+L+n+l-S_{z}+1\right), \\
& \Psi_{N L n l}^{S, S_{z}}(\zeta, \Phi, \xi, \phi)=\frac{1}{2 \pi} \mathrm{e}^{\mathrm{i} L \Phi} \mathrm{e}^{\mathrm{i} l \phi} U_{N L}(\zeta) u_{n l}(\xi) \chi\left(S, S_{z}\right)
\end{aligned}
$$

with

$$
U_{N L}(\zeta)=\frac{1}{R_{H} \sqrt{N !(N+L) !}} \mathrm{e}^{-\frac{\zeta}{2}} \zeta^{\frac{L}{2}} L_{N}^{L}(\zeta),
$$


and

$$
u_{n l}(\xi)=\frac{1}{r_{H} \sqrt{n !(n+l) !}} \mathrm{e}^{-\frac{\xi}{2}} \xi^{\frac{l}{2}} L_{n}^{l}(\xi),
$$

where $N, L$ and $n, l$ characterize respectively the orbital $(N, n)$ and angular-momentum $(L, l)$ quantum numbers for the center-of-mass and the relative motion. The part $\chi\left(S, S_{z}\right)$ represents the spin part of the wave function. Finally, the dimensionless coordinates have been defined

$$
\zeta=\frac{R^{2}}{2 R_{H}^{2}} \text { with } R_{H}^{2}=\frac{\hbar}{M \omega_{\mathrm{c}}} \text {, and } \xi=\frac{r^{2}}{2 r_{H}^{2}} \text { with } r_{H}^{2}=\frac{\hbar}{\mu \omega_{\mathrm{c}}} \text {. }
$$

The parameters $R_{H}$ and $r_{H}$ are the cyclotron radii of the two parts: the center of mass and that of orbiting one electron against the other.

A very important feature of the problem should be noted. Namely, the wave function parity is defined by the factor $(-1)^{l}(-1)^{S+1}$. Due to the Pauli principle the total wave function must have an odd parity. Let us note that the quantum number $L$ does not appear in the parity as the change $\boldsymbol{r}_{1} \leftrightarrow \boldsymbol{r}_{2}$ does not affect $\boldsymbol{R}$, but it does change the sign of $\boldsymbol{r}$. Also, for a noninteracting system the state with $l=1$ and $S_{z}=0$ are degenerate with that having $l=0$ and $S_{z}=1$ (the lowest singlet and triplet states, respectively).

\subsection{Real-space bound Cooper pair for $B \neq 0$}

One may think that, in direct analogy to the original Cooper situation [1], an extension of the solution to the situation with $V(r) \neq 0$ requires taking into account the following superposition of the previous wave functions:

$$
\Phi_{N L l}^{S, S_{z}}(R, \Phi, r, \phi)=\sum_{n} \alpha_{n} \Psi_{N L n l}^{S, S_{z}}[\zeta(R), \Phi, \xi(r), \phi] .
$$

However, this is not the case, as the Landau-type wave function does not reflect properly the situation in the $B \rightarrow 0$ limit (the cyclotron radii $R_{H}$ and $r_{H}$ diverge then). This singularity, which is also present when considering Landau orbits in the $B \rightarrow 0$ for single electrons, makes the problem highly nontrivial.

We propose the following variational approach for calculating the ground-state energy for the spin-singlet state in an arbitrary field. Namely, the ground-state wave function is taken in the form

$$
\Psi(r, R)=\frac{1}{\sqrt{2 \pi} \alpha} \mathrm{e}^{-r^{2} / \alpha^{2}} \frac{1}{\sqrt{2 \pi} \eta} \mathrm{e}^{-R^{2} / \eta^{2}},
$$

where $\alpha$ and $\eta$ are the variational parameters. Substituting this wave function into (4) and evaluating $\langle\Psi|H| \Psi\rangle$ we obtain the equation

$$
\begin{aligned}
E= & E(\alpha, \eta)=\frac{M \omega_{\mathrm{c}}^{2} \eta^{2}}{4}+\frac{\hbar^{2}}{4 M \eta^{2}}+\frac{\mu \omega_{\mathrm{c}}^{2} \alpha^{2}}{4}+\frac{\hbar^{2}}{4 \mu \alpha^{2}}-\hbar \omega_{\mathrm{c}} S_{z} \\
& -V_{0}\left(1-\mathrm{e}^{-a^{2} / 2 \alpha^{2}}\right) .
\end{aligned}
$$

Minimizing this equation with respect to $\eta$, we obtain $\eta_{\min }=R_{H}$, as it should be, since the center of mass motion is not influenced by the pairing interaction. Substituting this result into (12) and defining the dimensionless variables 


$$
\beta \equiv \frac{B}{B_{0}}, \quad \lambda \equiv \frac{\alpha}{a}, \quad \text { and } \quad \chi \equiv \frac{V_{0} m a^{2}}{2 \hbar^{2}},
$$

with $B_{0} \equiv m V_{0} / \hbar e$, we arrive at

$$
\frac{E\left(\lambda, R_{H}\right)}{V_{0}}=\frac{\beta}{2}+\frac{\lambda^{2}}{4} \chi \beta^{2}+\frac{1}{4 \lambda^{2} \chi}-\left(1-\mathrm{e}^{-1 / 2 \lambda^{2}}\right) .
$$

Minimizing this expression with respect to $\lambda$ for different values of $\beta$ and fixed value of $\chi$, we determine the pair binding energy defined as

$$
\tilde{\Delta}_{L=0, l=0}^{S=0, S_{z}=0}=E_{\min }\left(\lambda, R_{H}\right)-\left(E_{0}\right)_{L=0, l=0}
$$

for the spin-singlet state and, accordingly, for other states. In Fig. 1 we plot the binding energy for the lowest solution taking $\chi=10$ and $a=100 \AA$, when thus $V_{0}=15 \mathrm{meV}$ and $B_{0}=130 \mathrm{~T}$.

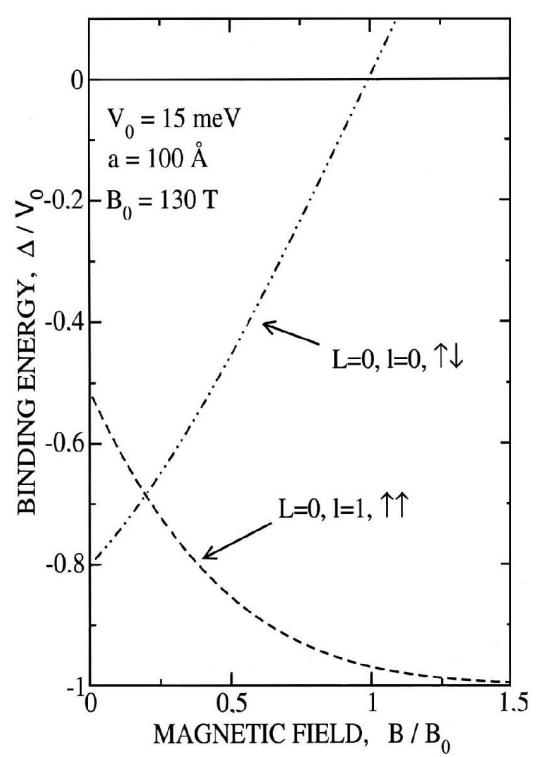

Fig. 1. Applied field dependence of pair binding energy for the spin singlet and triplet pairs in a two-dimensional attractive potential well for the pair.

Repeating the same procedure for the spin-triplet $\left(S_{z}=l=1\right)$ state, we have

$$
\Psi(r, R, \Phi)=\frac{1}{\sqrt{2 \pi} \eta} \mathrm{e}^{-R^{2} / 4 \eta^{2}} \frac{r}{2 \sqrt{\pi} \alpha^{2}} \mathrm{e}^{\mathrm{i} \Phi} \mathrm{e}^{-r^{2} / 4 \alpha^{2}}
$$

and correspondingly

$$
\frac{E\left(\lambda, R_{H}\right)}{E_{0}}=\frac{\lambda^{2}}{2} \chi \beta^{2}+\frac{1}{2 \lambda^{2} \chi}-\left[1-\mathrm{e}^{-1 / 2 \lambda^{2}}\left(1+\frac{1}{2 \lambda^{2}}\right)\right] .
$$

The binding energy in this case $\Delta_{L=1, l=0}^{S=1, S_{z}=1} \equiv E_{\min }(\lambda, \eta)-\left(E_{0}\right)_{L=1, l=0}$ has also been plotted in Fig. 1. As one can expect, the spin-triplet state becomes stable in strong applied field. 
One feature of the solution should be mentioned. Namely, there exists a critical value of $\chi$, below which the bound state ceases the existence. This is because of the confining potential existing only in the limited region of the size $a$, since it produces zero-point kinetic energy of the pair. This critical value of the potential is determined from the condition $\Delta=0$.

\subsection{Effect of the Coulomb repulsion on the pair binding}

To examine the effect of the Coulomb repulsion we consider two types of the interaction potential

$$
\text { 1) } V_{\text {rep }}=U \frac{a}{r}, \text { 2) } V_{\text {rep }}=U a^{2} \delta(\boldsymbol{r}) \text {. }
$$

The first case with $U \equiv e^{2} / \kappa a$, where $\kappa$ is the static dielectric constant, describes the long-range nature of the interaction. The second case expresses a well screened interaction.

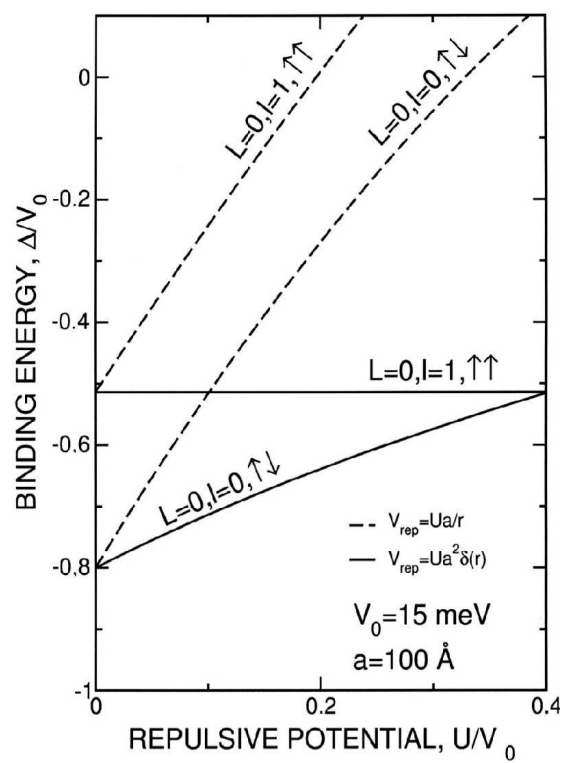

Fig. 2. Effect of the Coulomb repulsion amplitude $U$ on the pair binding energy. The two forms of repulsive Coulomb interaction are specified.

To calculate the system energy in the present situation, we start from the total wave function (11) with $\eta=R_{H}$ and minimize it with respect to $\alpha$. First, in Fig. 2 we plot the binding energy for the two cases and the corresponding states when $B=0$ (the center-of-mass part of the wave function is ignored then). The spin-singlet state is stable for weak repulsive interaction. The combined effect of the repulsive interaction and the applied field is shown in Fig. 3. The short-range type interaction stabilizes always the spin-triplet cases, whereas for the long-range potential it is stable above a threshold $B_{\mathrm{C}} \sim 0.3 B_{0}$. 


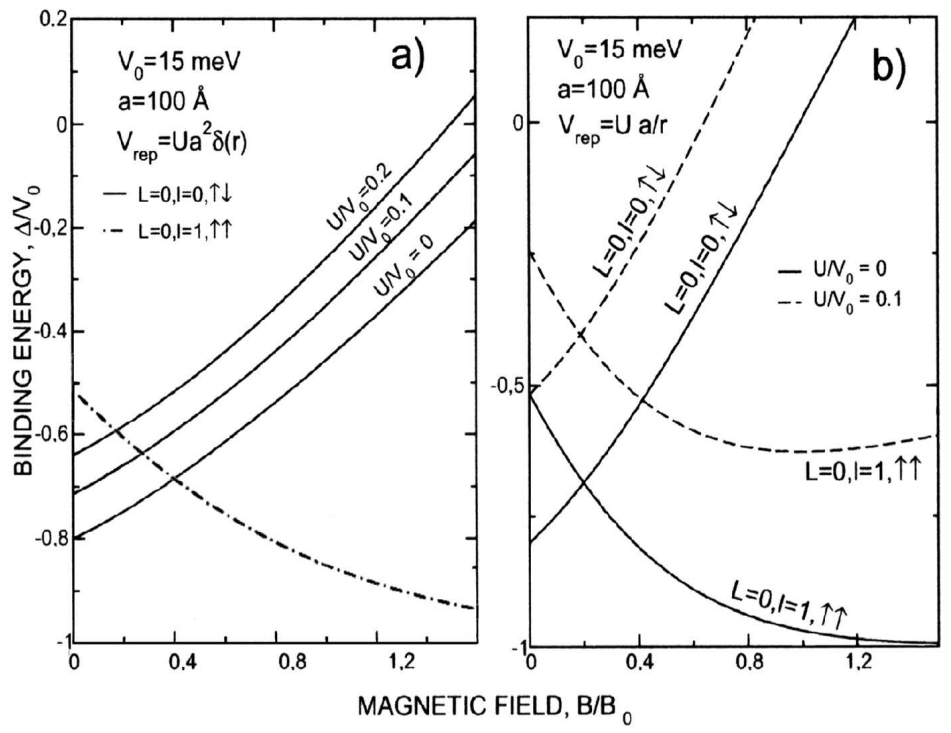

Fig. 3. Applied field dependence of pair binding energy for the short-range (a) and long-range (b) repulsive Coulomb interaction between electrons.

In conclusion, a singlet-triplet transition for the bound pair is possible in a rather strong applied field even if the pairing potential is spin independent. It would be interesting to see if there exists a type-II superconductor for which $B_{\mathrm{C}}<B_{\mathrm{C} 2}$. There are claims [7] that this happens in $\mathrm{Na}_{x} \mathrm{CoO}_{2}$ superconductor.

\section{Cooper pair with spin-dependent masses}

The spin-split masses for quasiparticles in the strongly correlated systems have been predicted theoretically [5] and recently detected experimentally in the heavy-fermion superconductor $\mathrm{CeCoIn}_{5}$ [8]. Here we consider a single pair with the spin-dependent masses. This means that we start from the Hamiltonian

$$
H=-\frac{\hbar^{2}}{2 m_{\uparrow}} \nabla_{1}^{2}-\frac{\hbar^{2}}{2 m_{\downarrow}} \nabla_{2}^{2}+V\left(\left|\boldsymbol{r}_{1}-\boldsymbol{r}_{2}\right|\right) .
$$

We introduce the usual form of the spatial part of the wave function [1]:

$$
\Psi\left(\boldsymbol{r}_{1}, \boldsymbol{r}_{2}\right)=\sum_{\boldsymbol{k}_{1} \boldsymbol{k}_{2}} \alpha_{\boldsymbol{k}_{1} \boldsymbol{k}_{2}} \frac{1}{V} \mathrm{e}^{\mathrm{i}\left(\boldsymbol{k}_{1} \cdot \boldsymbol{r}_{1}+\boldsymbol{k}_{2} \cdot \boldsymbol{r}_{2}\right)} .
$$

Next, we introduce the center-of-mass and relative coordinates

$$
\left\{\begin{array}{l}
\boldsymbol{R}=\frac{m_{\uparrow} \boldsymbol{r}_{1}+m_{\downarrow} \boldsymbol{r}_{2}}{m_{\uparrow}+m_{\downarrow}} \\
\boldsymbol{r}=\boldsymbol{r}_{1}-\boldsymbol{r}_{2}
\end{array}, \quad\left\{\begin{array}{l}
\boldsymbol{K}=\boldsymbol{k}_{1}+\boldsymbol{k}_{2} \\
\boldsymbol{k}=\frac{\boldsymbol{k}_{1} m_{\downarrow}-\boldsymbol{k}_{2} m_{\uparrow}}{m_{\uparrow}+m_{\downarrow}}
\end{array} .\right.\right.
$$

Let us note that the relative momentum is introduced in a nonstandard manner. In the new representation the further calculations are standard, so we will write down only the condition for the pair ground-state energy $E$ which is 


$$
1=\frac{1}{V} \sum_{\boldsymbol{k}} \frac{V_{0}}{\frac{\hbar^{2} \boldsymbol{k}^{2}}{2 \mu}-E}
$$

The integration over $\boldsymbol{k}$ is nonstandard, as illustrated in Fig. 4. Namely, the interval of nonzero pairing potential is contained between $k_{\mathrm{F} \downarrow} \leq k \leq k_{a \uparrow}$, where $k_{a \uparrow}$

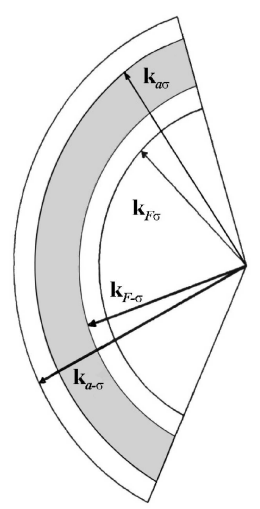

Fig. 4. The range of integration over $k$ for both spin directions $\sigma=\downarrow$ and $-\sigma=\downarrow$. For details see main text.

determines the maximal momentum transfer due to phonons; we take it as $k_{a \sigma}=$ $k_{\mathrm{F} \sigma}+\Delta k$. Under this circumstance, Eq. (22) reads

$$
1=\frac{V_{0}}{2 \pi} \int_{k_{\mathrm{F} \downarrow}}^{k_{a \uparrow}} \mathrm{d} k \frac{k^{2}}{\frac{\hbar^{2} \boldsymbol{k}^{2}}{2 \mu}-E} .
$$

As the integration integral is narrow, we make an approximation $k^{2} \approx \overline{\boldsymbol{k}}_{\mathrm{F}} k$, with $\overline{\boldsymbol{k}}_{\mathrm{F}} \equiv\left(k_{\mathrm{F} \uparrow}+k_{\mathrm{F} \downarrow}\right) / 2$. In effect, the expression for the binding energy can be brought up to the closed form [9]:

$$
\Delta=\frac{1}{\exp \left(\frac{2 \pi^{2} \hbar^{2}}{\mu \overline{\boldsymbol{k}}_{\mathrm{F}} V_{0}}\right)-1}\left\{\frac{m_{\uparrow}}{\mu} \hbar \omega_{\mathrm{D}}-\Delta m \epsilon_{\mathrm{F}}\left[\frac{1}{m_{\downarrow}}+\frac{1}{m_{\uparrow}} \exp \left(\frac{2 \pi^{2} \hbar^{2}}{\mu \overline{\boldsymbol{k}}_{\mathrm{F}} V_{0}}\right)\right]\right\},
$$

where $\hbar \omega_{\mathrm{D}}$ is the typical phonon frequency, $\Delta m=m_{\downarrow}-m_{\uparrow}$, and $\epsilon_{\mathrm{F}}$ is the Fermi energy. This result reduces to the Cooper result [1] when $\Delta m=0$. The binding energy decreases with the increasing $\Delta m$.

In conclusion, in the situation with spin masses the relative wave vector is defined differently. Also, the expression for the pair binding energy differs when the triplet pairing takes place [10]. The details of this analysis will be presented elsewhere.

\section{Concluding remarks}

The singlet Cooper-pair is destroyed by sufficiently strong applied field. This is not the case for the spin-triplet Cooper pair. In the latter case, the spin-split masses introduce inequivalence between the gap components $\Delta_{\uparrow \uparrow}, \Delta_{\downarrow \downarrow}$, and $\Delta_{\uparrow \downarrow}$, 
and a change of behavior of the spin-triplet superconductor or superfluid in an applied magnetic field.

\section{Acknowledgments}

The work was supported by both Ministry of Education and Science and by the Foundation for Polish Science (FNP).

\section{References}

[1] L.N. Cooper, Phys. Rev. 104, 1189 (1956). See also: M. Randeria, J.-M. Duan, L.-Y. Shieh, Phys. Rev. B 41, 327 (1990).

[2] J. Bardeen, L.N. Cooper, J.R. Schrieffer, Phys. Rev. 108, 1175 (1957).

[3] A single pair in a vacuum is discussed in: K. Byczuk, J. Spałek, W. Wójcik, Phys. Rev. B 46, 14134 (1992).

[4] M. Ortanõ, E. Cuevas, Europhys. Lett. 46, 224 (1999); G. Benedetti, D.L. Shepelyansky, cond-mat/0011461.

[5] J. Spałek, P. Gopalan, Phys. Rev. Lett. 64, 2823 (1990); P. Korbel, J. Spałek, W. Wójcik, M. Acquarone, Phys. Rev. B 52, R2213 (1995).

[6] For the case of single electron see e.g. L.D. Landau, E.M. Lifshitz, Quantum Mechanics, Pergamon Press, Oxford 1980, Par. 112; cf. also A.B. Pipard, The Physics of Vibration, Cambridge University Press, Cambridge 1983, Ch. 15.

[7] M. Maśka, M. Mierzejewski, B. Andrzejewski, M.L. Foo, R.J. Cava, T. Klimczuk, Phys. Rev. B 70, 144516 (2004).

[8] A. McCollam, S.R. Julian, P.M.C. Rourke, D. Aoki, J. Flouquet, Phys. Rev. Lett. 94, 186401 (2005).

[9] P. Wróbel, M.Sc. thesis, Jagiellonian University, Kraków 1999, unpublished.

[10] Z. Starypan, M.Sc. thesis, Jagiellonian University, Kraków 2004, unpublished. 